\title{
Selective reduction of nitro group using CuNi bimetallic nanoparticles
}

\author{
Mompiya Sanyal $^{1}{ }^{10} \cdot$ Uma Sharma $^{1}$
}

(c) Springer Nature Switzerland AG 2019

\begin{abstract}
Bimetallic nanoparticles have shown a lot of promise as catalysts in the recent years. This piece of work highlights the use of nanocatalyst for the chemoselective reduction of nitro group in presence of other reducible functional groups. CuNi bimetallic nanoparticles of varying composition $(1: 1,1: 2)$ have been synthesized by polyol method using metal sulphates with hydrazine hydrate as reducing agent. The characterization of synthesised nanoparticles was done by XRD and FESEM with EDX, which confirm FCC structure and composition of synthesised bimetallic nanoparticles. Particle size varies with composition i.e. $23 \mathrm{~nm}$ for (1:1) and $20 \mathrm{~nm}$ for (1:2). The selective reduction of $p$-nitrophenol, $p$-nitrobenzaldehyde and 2,4 dinitrophenol was performed in water using both single Ni nanoparticles and bimetallic CuNi nanoparticles $(1: 1,1: 2)$ with hydrazine hydrate as reducing agent at room temperature. The various amino products obtained has various industrial applications. UV-visible spectroscopy was used to monitor the progress of the reaction. The bimetallic nanoparticles facilitated the rate of reaction much as compared to single Ni nanoparticles.
\end{abstract}

\section{Graphical abstract}

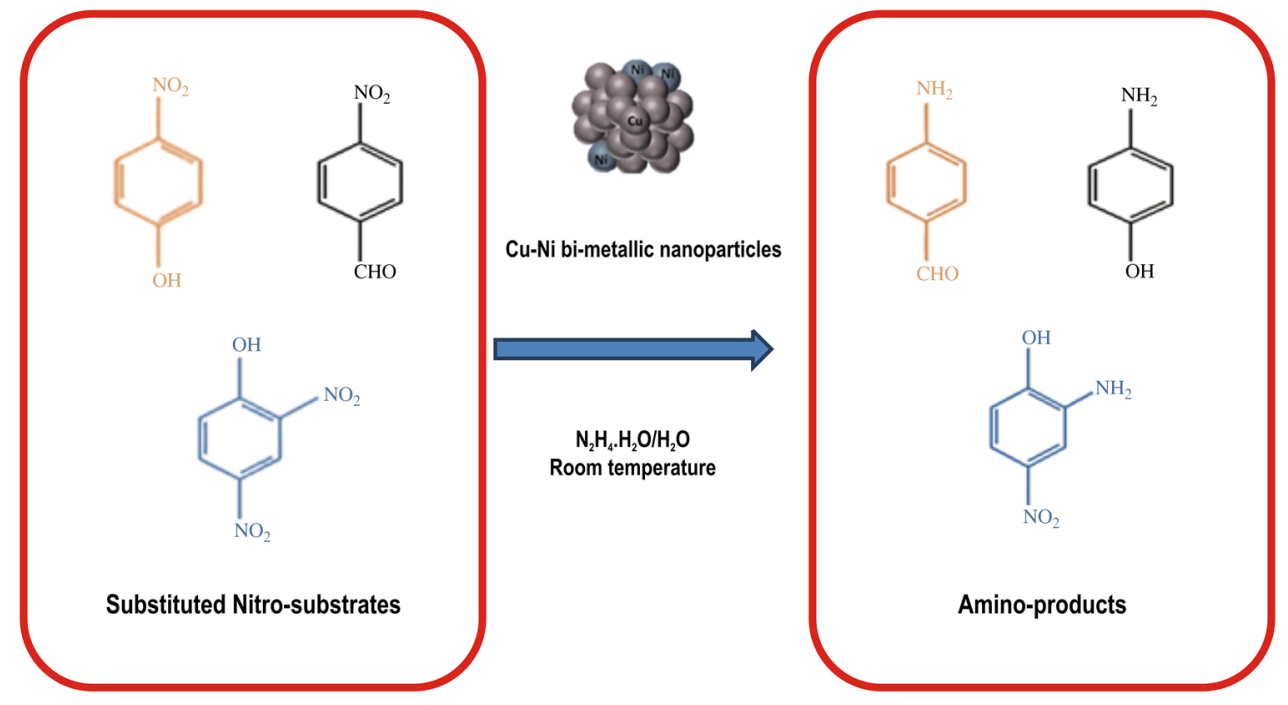

Keywords Bimetallic · Polyol · Synergistic · Nanocatalyst

Mompiya Sanyal, mompiyasanyal@gmail.com; Uma Sharma, umasharma10@rediffmail.com | 'School of Studies in Chemistry and Biochemistry, Vikram University, Ujjain, M.P. 456010, India. 


\section{Introduction}

Nitrosubstituted aromatic hydrocarbons are generally toxic and very difficult to be degraded in the environment. The conversion of aromatic nitro compounds to aromatic amino compounds obey with the demands of green chemistry [1]. Para nitrophenol and its derivatives are used to manufacture herbicides, insecticides and synthetic dyestuffs and they can substantially damage the ecosystem along with the common organic pollutants [2]. They are harmful for our health as they can damage the liver, kidney, central nervous system. Therefore removal of nitrophenol and its derivatives is very necessary. The reaction product 4-aminophenol and its derivatives are useful compounds which are used as intermediates for manufacturing analgesics, antipyretics, photographic developer, anti-corrosion lubricant etc. [3].

Nanomaterials are the cornerstones of nanoscience and technology. The potential applications of nanomaterials are in nanocatalysis [4], nanomedicine [5], chemical and biosensors [6], and in drug delivery [7]. In this context, metal nanoparticles based on noble metals ( $\mathrm{Pt}, \mathrm{Pd}, \mathrm{Au}$ etc.) have shown high potential towards the reduction of nitro groups using $\mathrm{H}_{2}, \mathrm{NaBH}_{4}, \mathrm{~N}_{2} \mathrm{H}_{4} \cdot \mathrm{H}_{2} \mathrm{O}$ etc. as reducing agents [8-15]. But the high cost of noble metals makes them undesirable to use them as catalyst, despite showing high selectivity. This paved the way for the development of inexpensive non noble metal based catalysts, which will undoubtedly be highly economical [16-23].

In this piece of work, the selective reduction of the nitro group into amino group has been carried out using nickel nanoparticles and bimetallic CuNi nanoparticles of different compositions $(1: 1,1: 2)$ with hydrazine hydrate as reducing agent using water as green solvent at room temperature. Bimetallic nanoparticles displayed enhanced catalytic activity as compared to pure nanoparticles due to synergistic effect [24].

1.

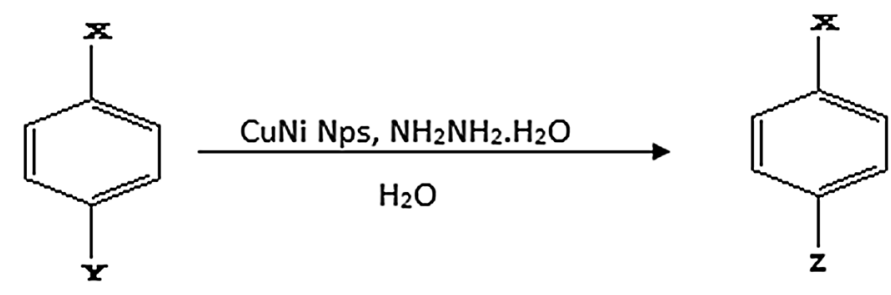

$$
\text { Where } \begin{aligned}
X & =\mathrm{OH} \\
Y & =\mathrm{NO}_{2}
\end{aligned}
$$

2.

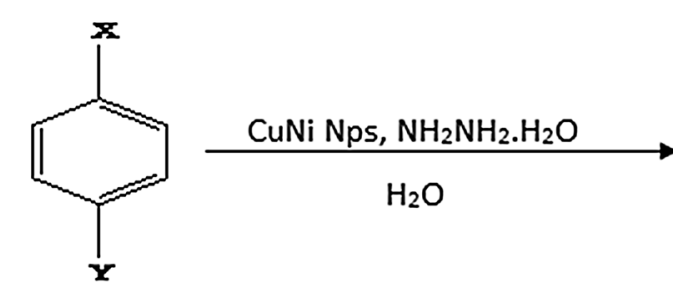

$$
\text { Where } \begin{aligned}
X & =\mathrm{CHO} \\
Y & =\mathrm{NU}_{2}
\end{aligned}
$$

3.<smiles>[X]c1ccc([X])c([Y])c1</smiles>

Where $X=\mathrm{OH}$

$\mathrm{Y}=\mathrm{NO}_{2}$
Where $\mathrm{X}=\mathrm{OH}$

$\mathrm{z}=\mathrm{NH}_{2}$
Where $X=\mathrm{CHO}$ $\mathrm{Z}=\mathrm{NH}_{2}$ 


\section{Experimental section}

Copper sulphate and nickel sulphate were purchased from $\mathrm{CDH}$, solvents were of analytical grade and used as received. $p$-nitrophenol, $p$-nitrobenzaldehyde and 2,4 dinitrophenol were purchased from Merck.

Powder X-ray diffraction (XRD) measurements were performed using Bruker D8 advance X-Ray diffractometer using CuKa radiation ( $\lambda=0.154 \mathrm{~nm}$ ) and the interpretation was done with by Origin software. FESEM images were taken using "FEI NoVa NanoSEM 450" FESEM instrument operated at $18 \mathrm{kV}$. Compositional analysis were made using "Bruker" made X-Flash 6130 EDS attachment and "Esprit" software. UV-visible spectral studies were performed using Perkin Elmer Lamda 25 UV-visible Spectrometer. IR spectral study was performed using Perkin Elmer Spectrum Version 10.5.1.

\subsection{Synthesis of CuNi bimetallic nanoparticles (1:1, 1:2) by polyol method}

$0.1 \mathrm{M}(0.2496 \mathrm{~g})$ copper sulphate and $0.1 \mathrm{M}(0.1547 \mathrm{~g})$ nickel sulphate (1:1)/0.2 M (0.3095) nickel sulphate (1:2) were dissolved in $50 \mathrm{~mL}$ of ethylene glycol, followed by addition of $3 \mathrm{~mL}$ hydrazine hydrate. Few drops of $1 \mathrm{M}$ $\mathrm{NaOH}$ was added with continuous stirring for maintaining $\mathrm{pH}=11.5$ and then the temperature of the reaction mixture was maintained at $180^{\circ} \mathrm{C}$ for $2 \mathrm{~h}$. The nanoparticle suspension was centrifuged at 10,000 rpm, washed with ethanol and dried at $60{ }^{\circ} \mathrm{C}$ overnight. Shiny green coloured crystals were obtained. Yield $=0.38 \mathrm{gm}$.

\subsection{General procedure for the reduction of nitro group using hydrazine hydrate at room temperature and CuNi $(1: 1,1: 2)$ nanoparticles as catalysts}

$5 \mathrm{mmol}$ of nitro substrate in $30 \mathrm{ml}$ distilled water was taken in a round bottom flask. To this $0.5 \mathrm{~mL}$ hydrazine hydrate was added and the reaction mixture was stirred for sometime at room temperature. After this $0.001 \mathrm{~g}$ of catalyst (synthesised nanoparticles) was added and the contents were stirred. The progress of the reaction was monitored every $3 \mathrm{~min}$ by UV-visible spectroscopy. After completion, the reaction mixture was centrifuged at $10,000 \mathrm{rpm}$ to separate out the catalyst [25].

\section{Results and discussion}

The XRD patterns of bimetallic CuNi nanoparticles with various ratios $(1: 1,1: 2)$ are shown in Fig. 1 . Both the samples possess FCC structure with the diffraction lines at (111), (200), (220), (311) planes. The average particle size decreases from $23 \mathrm{~nm}$ to $20 \mathrm{~nm}$ on increasing the nickel content. The lattice parameter (a) is found to decrease with increase in Ni content from 3.602 to 3.580 . The lattice parameters of bimetallic nanoparticles are located in between the lattice parameter of pure nickel i.e. 3.517

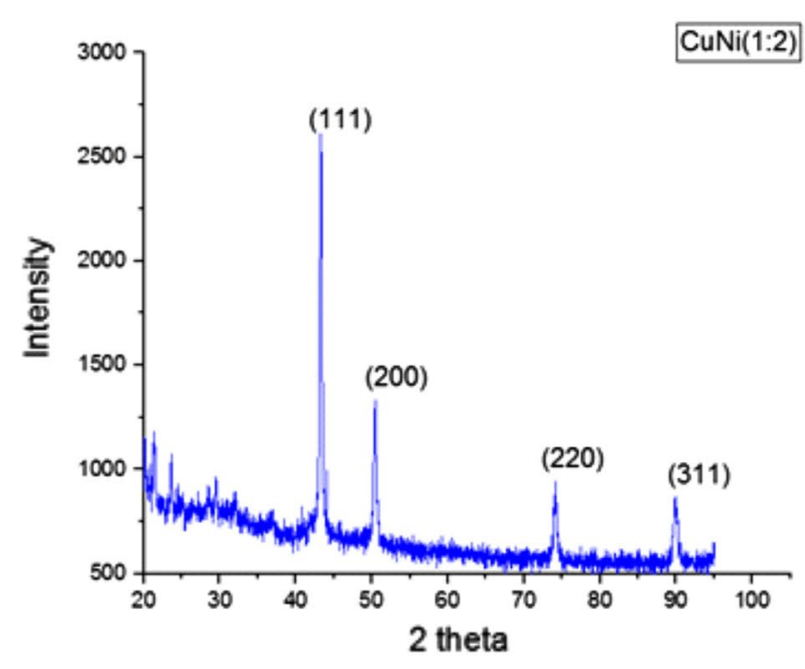

(b)

Fig. 1 a XRD of CuNi nanoparticles (1:1), b XRD of CuNi nanoparticles (1:2) 
Fig. 2 a FESEM images of CuNi (1:1) nanoparticles, b FESEM images of CuNi (1:2) nanoparticles

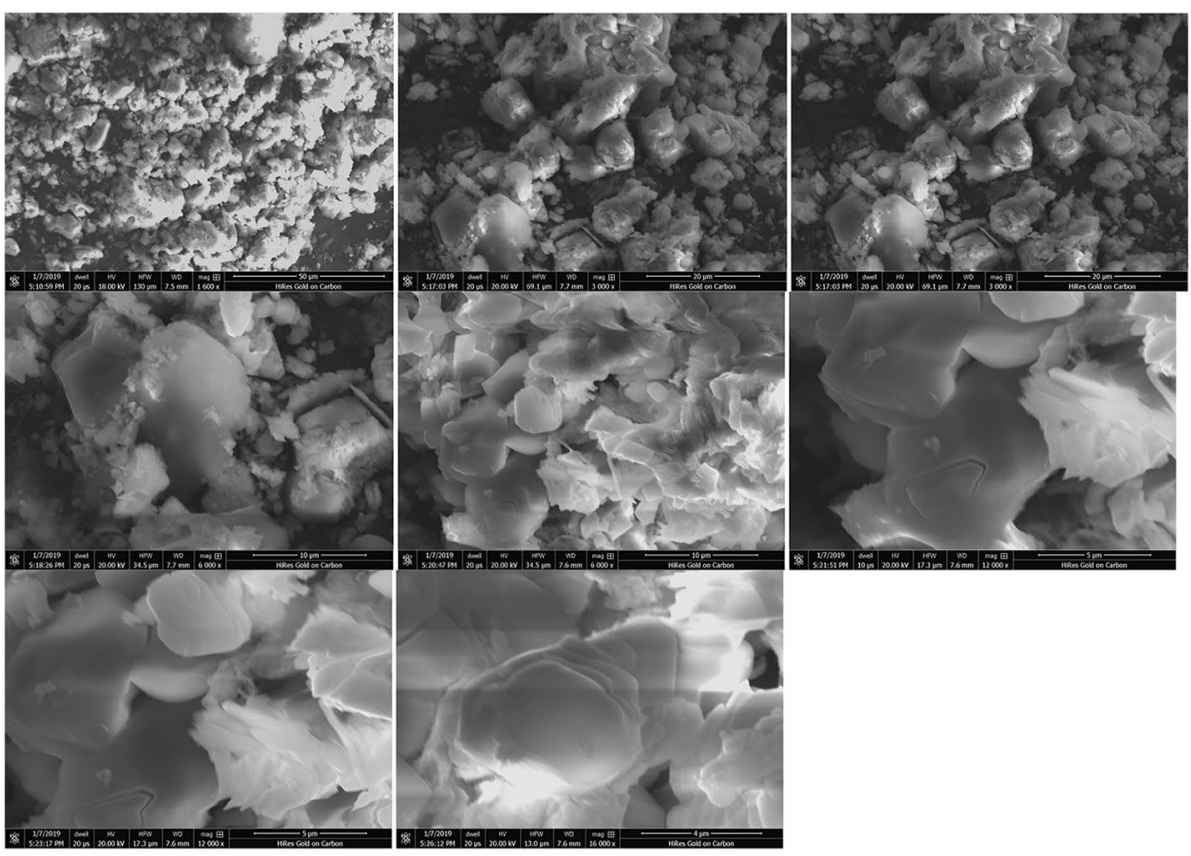

(a)

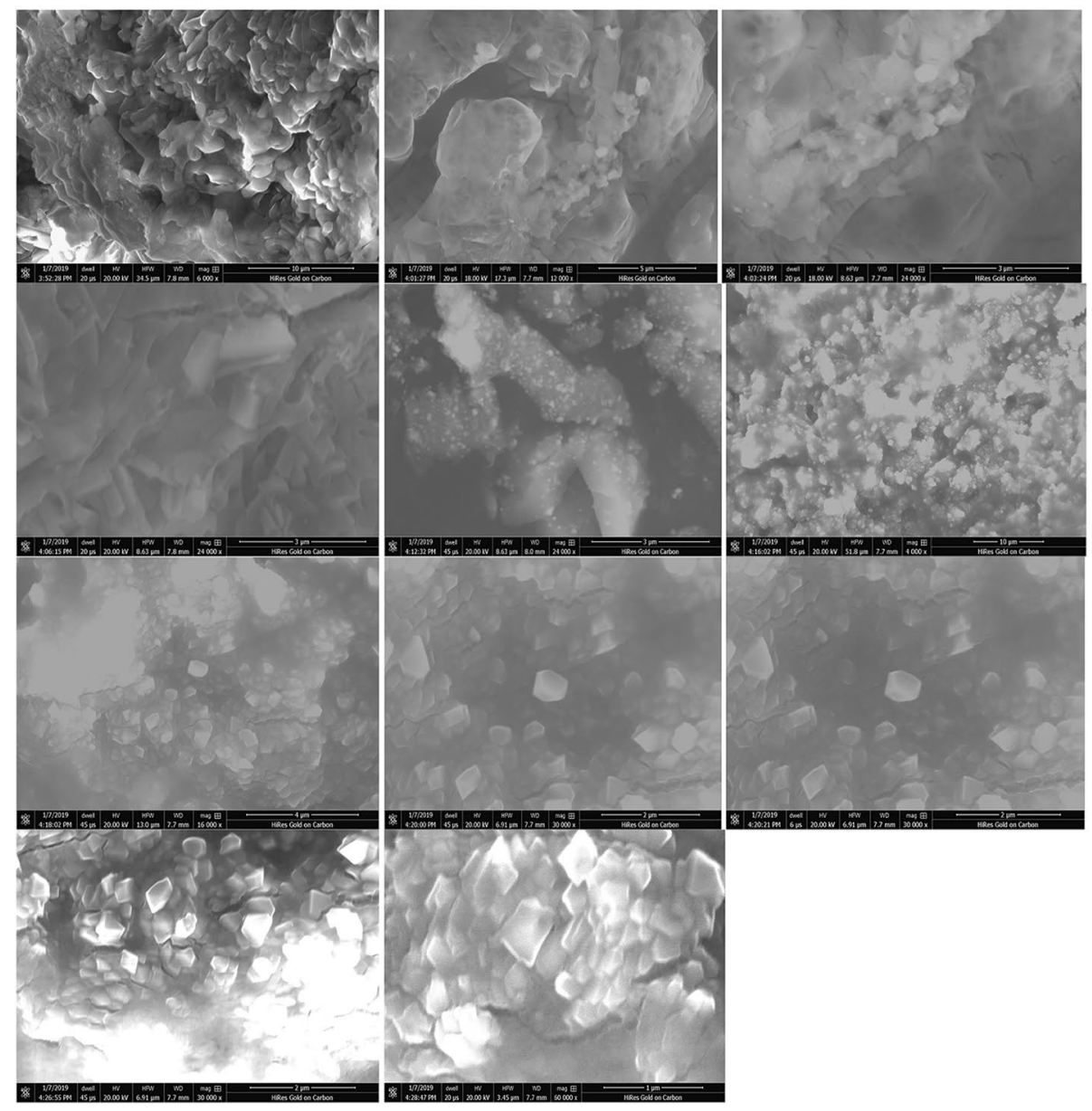

(b) 


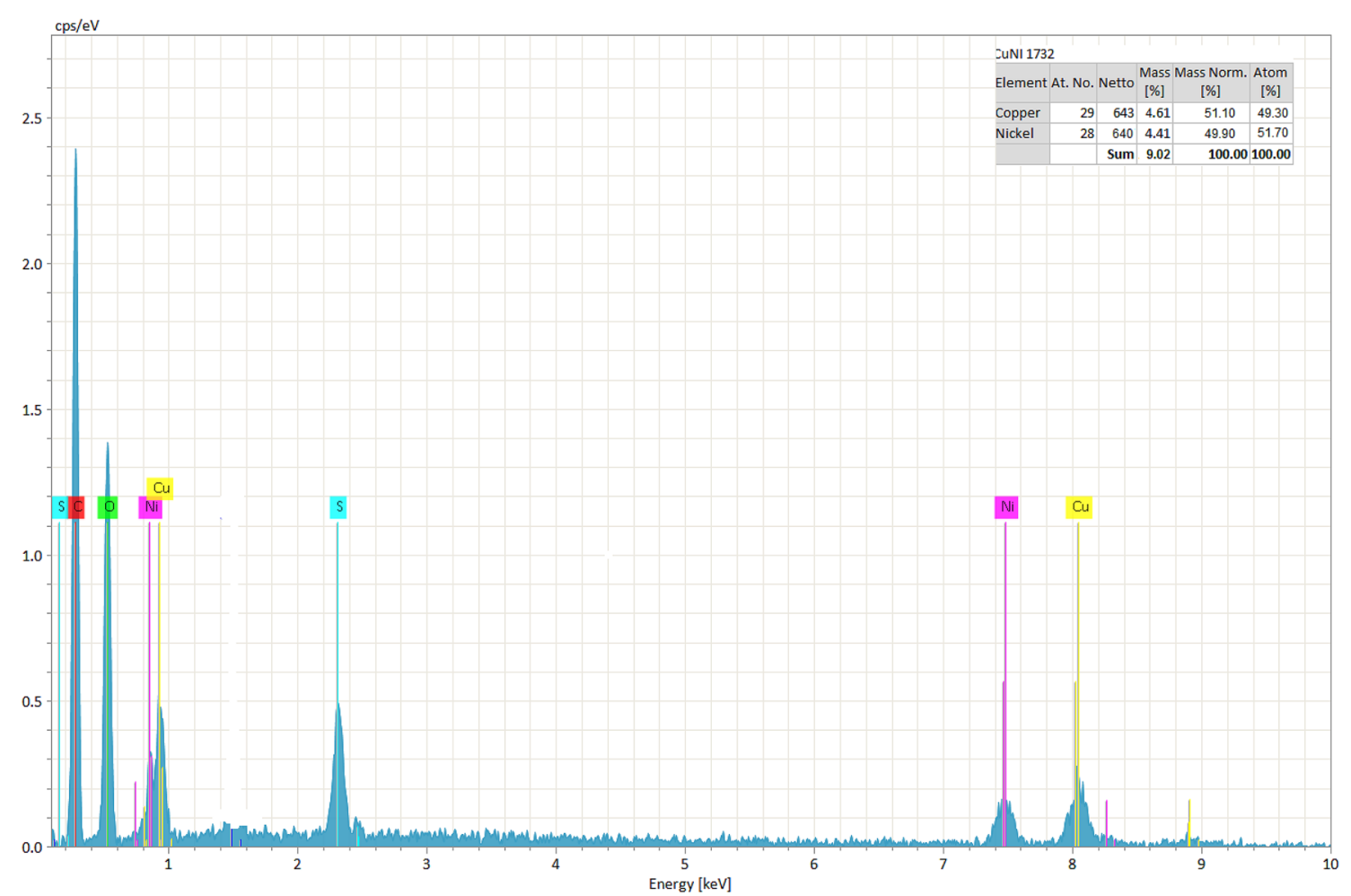

(a)

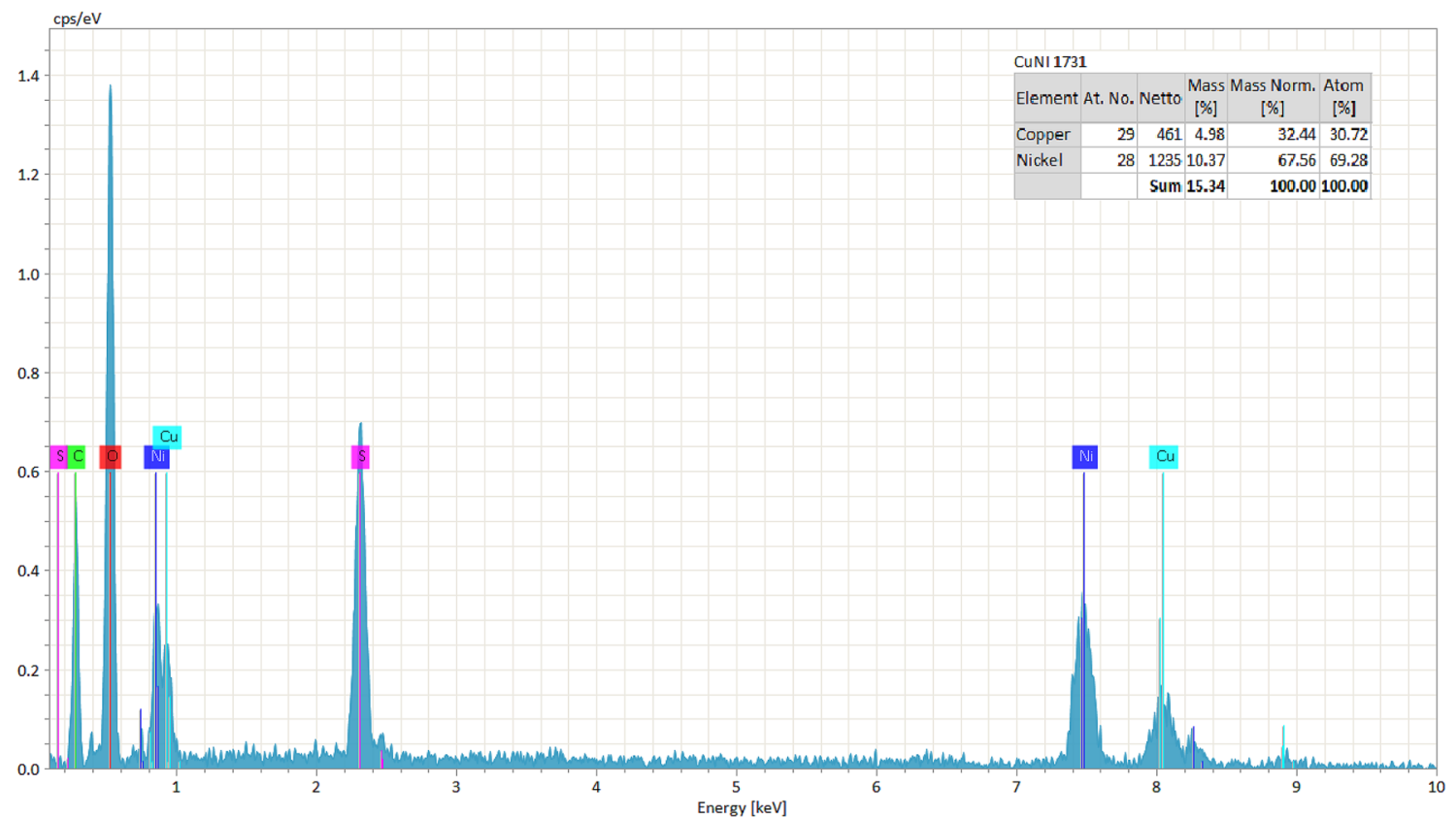

(b)

Fig. 3 a EDX spectrum of CuNi (1:1) nanoparticle, b EDX spectrum of CuNi (1:2) nanoparticle 
Table 1 Catalytic reduction of substituted nitro benzenes to the corresponding anilines

\begin{tabular}{|c|c|c|c|c|c|}
\hline $\begin{array}{l}\text { S. } \\
\text { no. }\end{array}$ & Substrate & Product & $\begin{array}{l}\text { Reaction } \\
\text { completion } \\
\text { time } \\
\text { without } \\
\text { catalyst }\end{array}$ & $\begin{array}{l}\text { Reaction } \\
\text { completion } \\
\text { time with } \\
\mathrm{Ni} \\
\text { nanoparticle }\end{array}$ & $\begin{array}{l}\text { Reaction } \\
\text { completion } \\
\text { time with CuNi } \\
\text { nanoparticle } \\
\left(1: 1^{*}, 1: 2^{* *}\right)\end{array}$ \\
\hline 1. & At basic medium & (absorbance peak at $310 \mathrm{~nm}$ ) & - & $45 \mathrm{~min}$ & $\begin{array}{l}30 \min ^{*} \\
20 \min ^{* *}\end{array}$ \\
\hline 2. & 267.43 nm, $217.93 \mathrm{~nm})$ & $313.95 \mathrm{~nm}, 214.8 \mathrm{~nm})$ & - & $30 \mathrm{~min}$ & $\begin{array}{l}15 \min * \\
8 \min ^{* *}\end{array}$ \\
\hline 3. & 2,4-dinitrophenol & 2-amino-4-nitrophenol & - & $75 \mathrm{~min}$ & $\begin{array}{l}45 \min ^{*} \\
30 \mathrm{~min} * *\end{array}$ \\
\hline
\end{tabular}

*Stands for the time taken to complete the reaction by 1:1 bimetallic nanoparticles

**Stands for time taken to complete the reaction time by 1:2 bimetallic nanoparticles

\section{SN Applied Sciences}

A SPRINGER NATURE journal 
(JCPDS 65-0380) and pure copper i.e. 3.615 (JCPDS 04-0836) which confirms the formation of bimetallic nanoparticles. With the increase in nickel content, the diffraction lines are shifted to high angle and more close to the position of pure nickel. There is a distinct peak at 110 plane $\left(2\right.$ theta $=37$ ) in Fig. 1 a which corresponds to $\mathrm{Cu}_{2} \mathrm{O}$ (Cuprite) confirmed using Match 3 software for XRD. No peaks corresponding to pure copper and pure nickel can be seen in the XRD pattern thereby excluding the possibility that the synthesised bimetallic nanoparticles are simply the mixtures of the two metal nanoparticles [26] (Fig. 2).

The synthesised CuNi bimetallic nanoparticles were further analysed by EDX (Energy Dispersive X-ray Analysis) and EDX spectrum are shown in Fig. $3 a$, b to confirm the formation and elemental composition of the nanoparticles. The elemental composition i.e. copper (49.30\%) and nickel (51.90\%) (Table in Fig. 3a) clearly indicates that 1:1 composition of CuNi bimetallic nanoparticles are present in sample 1. Similarly, the elemental composition i.e. copper (30.72) and nickel (69.28) (Table in Fig. 3a) clearly indicates that 1:2 composition of CuNi bimetallic nanoparticles are present in sample 2 .

The catalytic activity of the synthesised nanoparticles was evaluated for the reduction of nitro groups in various substrates using hydrazine hydrate as reducing agent at room temperature under aerobic conditions (Table 1).

The efficacies for the conversion of nitro group to amino group was studied for the model nitro substrate $p$-nitrophenol using the synthesised $\mathrm{Ni}, \mathrm{CuNi}(1: 1,1: 2)$ nanoparticles. The catalytic reaction was monitored by UV-visible spectroscopy (as shown in Fig. 4) where a gradual decrease in the intensity of the characteristic absorbance peak of

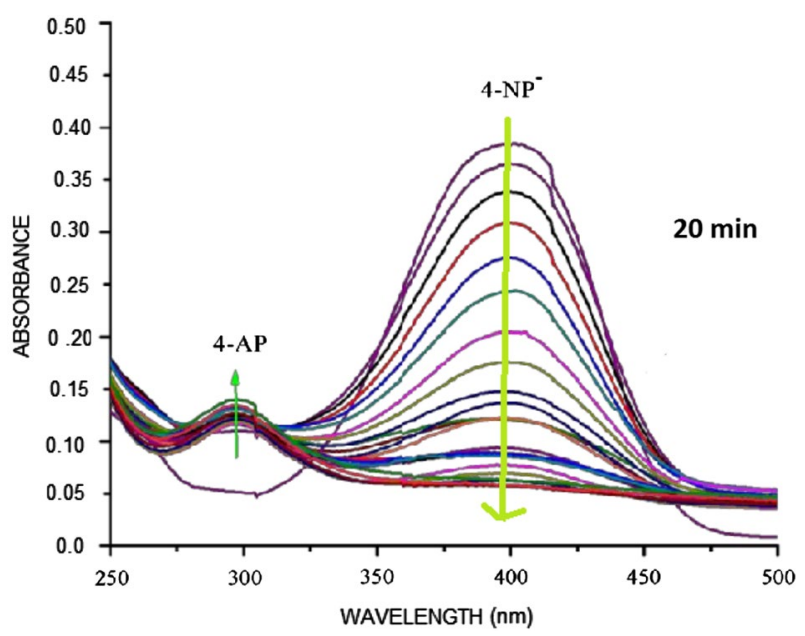

Fig. 4 UV-visible spectrum showing catalytic activity of CuNi (1:2) in case of $p$-Nitrophenol p-nitrophenol at $405 \mathrm{~nm}$ was observed along with the development of new absorbance peak at $310 \mathrm{~nm}$ which corresponds to the formation of $p$-amino phenol which has wide range of applications in photographic development of white and black films, drying agents, antipyretic and analgesic drugs and specially an important constituent of paracetamol. The reaction was completed in $45 \mathrm{~min}$ with $\mathrm{Ni}$ Nanoparticles, 30 min with CuNi Nanoparticles (1:1) and in 20 min with CuNi Nanoparticles (1:2). The reaction did not proceed either in the absence of catalyst or in the absence of hydrazine hydrate. We have performed experiment in water only without the addition of any organic solvent which is the most desirable condition. This finding can also be used to selectively reduce nitro group in those drugs which share the same basic structure, but differ only in their substituent groups as in case of benzodiazepines [27].

The synthesised nanocatalyst showed excellent chemoselectivity for the reduction of nitro group in $p$-nitrobenzaldehyde even in the presence of other reducible group. (i.e. $\mathrm{CHO}$ ) to $p$-aminobenzaldehyde. The absorbance at $267 \mathrm{~nm}$ which was due to nitro group gradually decreased with time and a new peak appear at $313.95 \mathrm{~nm}$ which was due to amino group. The reaction was completed in 15 min with CuNi nanoparticles (1:1) and in 8 min with CuNi nanoparticles (1:2) using hydrazine hydrate as reducing agent.

In case of 2,4-dinitrophenol, the synthesised bimetallic nanoparticles showed selective reduction of only one nitro group which can be a desirable product in any reaction. The UV spectrum of 2, 4 dinitrophenol shows the absorbance at $245 \mathrm{~nm}$ and $358 \mathrm{~nm}$ which is due to $\mathrm{OH}$ and $\mathrm{NO}_{2}$ group respectively. But with due course of time with the use of reducing agent and nanocatalyst, a new absorbance peak at $383 \mathrm{~nm}$ was seen which was due to $\mathrm{NH}_{2}$ group along with the presence of absorbance peak at $353 \mathrm{~nm}$. The results indicate that reduction of only one nitro group occurred. The reaction was completed in 75 min with $\mathrm{Ni}$ Nanoparticles, 45 min with CuNi Nanoparticles (1:1) and in 30 min with CuNi Nanoparticles (1:2). The results show the role of bimetallic nanoparticles to facilitate the reaction.

The identification of final product was done by melting point determination and IR spectrum. The melting point of the product is $127^{\circ} \mathrm{C}$ which corresponds to 4-amino-2-nitrophenol $\left(125-127^{\circ} \mathrm{C}\right)$. In IR spectrum (Fig. 5) broad peak observed at $3193.15 \mathrm{~cm}^{-1}$ is assigned to hydrogen bonded phenolic group i.e. nitro group at ortho position to phenolic group forms strong hydrogen bond, which confirms the retention of nitro group at ortho position. Hence, regioselective reduction at para position is confirmed.

The results indicate that the efficiency of $\mathrm{CuNi}$ $(1: 2)$ bimetallic nanoparticles as catalyst is more in comparison to $\mathrm{CuNi}(1: 1)$ nanaoparticles and pure $\mathrm{Ni}$ 
Fig. 5 IR spectrum of 4-amino2-nitrophenol
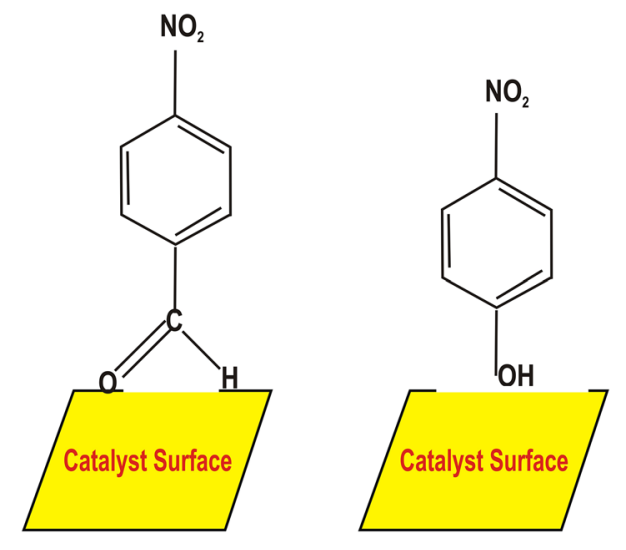

Fig. 6 Pictorial representation showing adherence of functional group to catalyst surface

nanoparticles. The catalytic activity enhanced in case of CuNi bimetallic nanoparticles (1:1, 1:2) as compared to pure nickel nanoparticles due to synergy between the two metals. The bimetallic CuNi Np of 1:2 composition facilitated the rate of reaction in all the substrates. This is due to increase in surface area of catalyst with decrease in average particle size of 1:2 bimetallic CuNi Nanoparticles. Greater the surface area of the catalyst, faster is the reaction.

Langmuir- Hinshelwood mechanism for heterogeneous catalysis advocates the adsorption of substrate and reducing agent on the surface of catalyst. The presence of nanoparticle facilitates the transfer of electron from reducing agent to the substrate [28].

As indicated in table the fastest reaction is observed with substrate containing $\mathrm{CHO}$ functional group which is electron withdrawing and simultaneously provide facile orientation to the substrate molecule in comparison to $\mathrm{OH}$ containing substrate (Fig. 6).

The mechanism of conversion of nitrosubstrates to amino products has been illustrated in Fig. 7 .

\section{Conclusion}

The CuNi bimetallic nanoparticles of different ratios $(1: 1,1: 2)$ have been synthesised successfully by polyol method with $93 \%$ yield. The XRD data confirms the face centred cubic arrangement of the synthesised nanoparticles and EDX analysis confirms the composition of the synthesised nanoparticles. The inexpensive non noble metal nanocatalyst displayed chemoselective reduction of range of nitro substrates into respective amines in the presence of other reducible functional groups. The efficiency of CuNi (1:2) bimetallic nanoparticles as catalyst is more in comparison to $\mathrm{CuNi}$ (1:1) nanaoparticles which is turn is more than pure Ni nanoparticles. The catalytic system is less expensive, easy to prepare, air stable, energy efficient and reproducible. Particle size and surface area of catalyst played a significant role in 

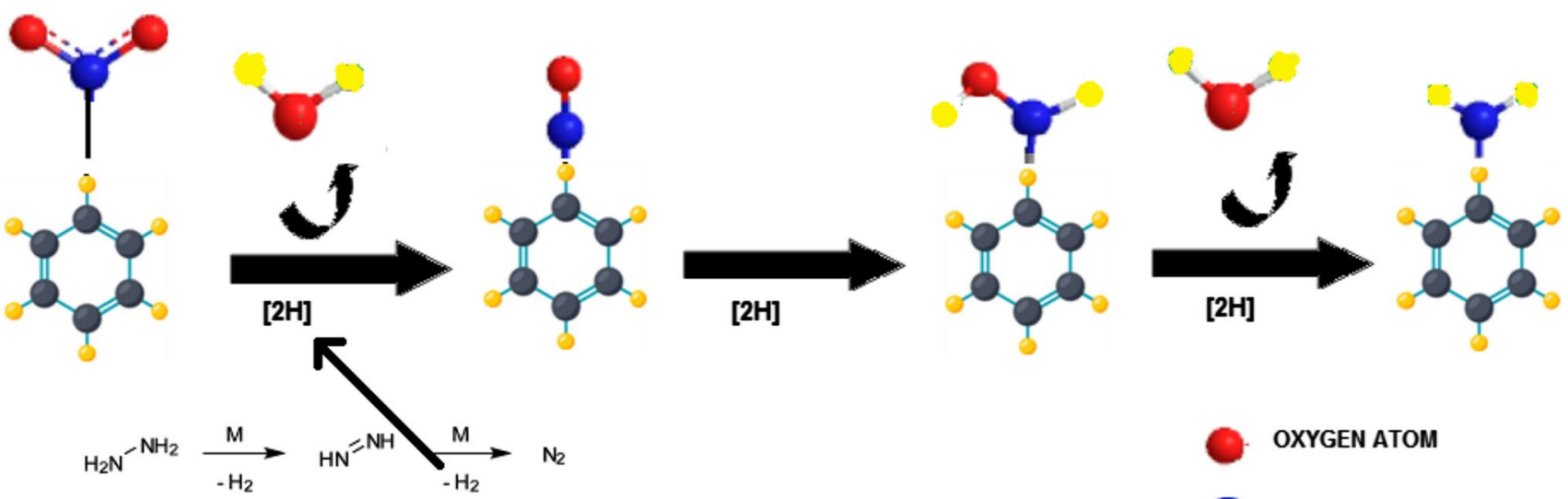

OXYGEN ATOM

NITROGEN ATOM

HYDROGEN ATOM

Fig. 7 Plausible mechanism for the catalytic reduction of nitro group

facilitating the rate of the reaction. Electrostatic interactions between catalyst surface and substrate can be monitored by selecting the substituent which provides more facile orientation thereby enhancing the rate of the reaction. All the catalytic reactions were performed at room temperature in aqueous medium-which is a greener route. Water and nitrogen are the only byproducts from the catalytic reaction which is a great advantage.

Acknowledgements We would like to thank the Centre-director, Dr. V. Ganeshan, UGC-DAE, Indore for allowing us to utilise available national facilities, Dr. M. Gupta, Dr. D. M. Phase and Dr. R. Venkatesh for providing XRD facilities, FESEM with EDX analysis respectively. Thanks to Prof. and Head, School of Studies in Chemistry and Biochemistry, Vikram University, Ujjain for providing research facilities.

\section{Compliance with ethical standards}

Conflict of interest On behalf of all authors, the corresponding author states that there is no conflict of interest.

\section{References}

1. Pradhan N, Pal A, Pal T (2002) Silver nanoparticle catalysed reduction of aromatic nitrocompounds. Colloids Surf A Physicochem Eng Asp 196:247-257

2. Gerelbaatar K, Tsogoo A, Tsedev N, Ganbold EO (2017) Reduction of 2,4 dinitrophenol to 2,4 diaminophenol using AuNanoparticles and AgNanoparticles as catalyst. Solid State Phenom 271:76-84

3. Ju KS, Parales RE (2010) Nitroaromatic compounds, from synthesis to biodegradation. Microbiol Mol Biol Rev 74(2):250-272

4. Braun T, Mark L, Ohmacht R, Sharma U (2007) Olive oil as a biocompatible solvent for pristine $\mathrm{C}_{60}$. Fuller Nanotub Carbon Nanostruct 15:311-314
5. Sharma S, Sharma U (2013) Synthesis, characterization and determination of encapsulation efficiency of chitosan nanoparticles for terbinafine. Indo Am J Pharm Res 3(12):1564

6. Zhang X, Guo Q, Cui D (2009) Recent advances in nanotechnology applied to biosensors. Sensors 9:1033-1053

7. Seleci M, Seleci DA, Joncyzk R, Stahl F, Blume C (2016) Smart multifunctional nanoparticles in nanomedicine. BioNanoMaterials $17(1-2): 33-41$

8. Corma A, Serna P (2006) Chemoselective hydrogenation of nitro compounds with supported gold catalysts. Science 313:332-334

9. Xiang $Y$, Meng Q, Li X, Wang J (2010) In situ hydrogen from aqueous-methanol for nitroarene reduction and imine formation over an $\mathrm{Au}-\mathrm{Pd} / \mathrm{Al}_{2} \mathrm{O}_{3}$ catalyst. J Chem Commun 46:5918-5920

10. Huang J, Yu L, He L, Liu Y-M, Cao Y, Fan K-N (2011) Direct imine formation by oxidative coupling of alcohols and amines using supported manganese oxides under an air atmosphere. Green Chem 13:2672-2677

11. Layek K, Kantam ML, Shirai M, Nishio-Hamane D, Sasaki T, Maheswaran H (2012) Gold nanoparticles stabilized on nanocrystalline magnesium oxide as an active catalyst for reduction of nitroarenes in aqueous medium at room temperature. Green Chem 14:3164-3174

12. Lin $X$, Wu M, Wu D, Kuga $S$, Endoe T, Huang $Y$ (2011) Platinum nanoparticles using wood nanomaterials: eco-friendly synthesis, shape control and catalytic activity for $p$-nitrophenol reduction. Green Chem 13:283-287

13. Luo P, Xu K, Zhang R, Huang L, Wang J, Xing W, Huang J (2012) Highly efficient and selective reduction of nitroarenes with hydrazine over supported rhodium nanoparticles. J Catal Sci Technol 2:301-304

14. Fan G, Huang W, Wang C (2013) In situ synthesis of Ru/RGO nanocomposites as a highly efficient catalyst for selective hydrogenation of halonitroaromatics. Nanoscale 5:6819-6825

15. Cai S, Duan H, Rong H, Wang D, Li L, He W, Li Y (2013) Highly active and selective catalysis of bimetallic $\mathrm{Rh}_{3} \mathrm{Ni}_{1}$ nanoparticles in the hydrogenation of nitroarenes. ACS Catal 3:608-612

16. Zhang N, Xu Y (2013) Aggregation- and leaching-resistant, reusable, and multifunctional $\mathrm{Pd} @ \mathrm{CeO}_{2}$ as a robust nanocatalyst achieved by a hollow core-shell strategy. J Chem Mater 25:1979-1988 
17. Chen Z, Liu S, Yang M-Q, Xu Y (2013) Aggregation- and leachingresistant, reusable, and multifunctional Pd@CeO 2 as a robust nanocatalyst achieved by a hollow core-shell strategy. J ACS Appl Mater Interfaces 1:1258-1266

18. Westerhaus FA, Jagadeesh RV, Wienhofer G, Pohl M-M, Radnik $J$, Surkus A-E, Rabeah J, Junge K, Junge $H$, Nielsen M, Bruckner A, Beller M (2013) Synthesis of uniform cds nanospheres/graphene hybrid nanocomposites and their application as visible light photocatalyst for selective reduction of nitro organics in water. Nat Chem 5:537-543

19. Vernekar AA, Patil S, Bhat C, Tilve SG (2013) Heterogenized cobalt oxide catalysts for nitroarene reduction by pyrolysis of molecularly defined complexes. RSC Adv 3:13243-13250

20. Saha A, Ranu B (2008) Magnetically recoverable catalytic Co$\mathrm{CO}_{2} \mathrm{~B}$ nanocomposites for the chemoselective reduction of aromatic nitro compounds. J Org Chem 73:6867-6870

21. Kadam HK, Tilve SG (2012) Convenient reduction of nitrobenzenes to anilines using electrochemically generated nickel. RSC Adv 2:6057-6060

22. Cantillo D, Baghbanzadeh M, Kappe CO (2012) Copper (II) bromide as a procatalyst for in situ preparation of active $\mathrm{Cu}$ nanoparticles for reductionof nitroarenes. Angew Chem Int Ed 51:10190-10193
23. Rezaei SJ, Malekzadeh AM, Ramazani A, Khorramabadi H (2018) Chemo-selective reduction of nitro and nitrile compounds using Ni nanoparticles immobilized on hyperbranched polymerfunctionalized magnetic nanoparticles. Appl Organomet Chem 32:3975-3987

24. Moganavally P, Suresh R, Deepa M (2015) Synthesis and characterization of bimetallic $\mathrm{CuNi}, \mathrm{CuNi}_{2}, \mathrm{CuNi}_{3}$ nanoparticles. Int J ChemTech Res 8(5):109-112

25. Rai RK, Mahata A, Gupta S, Nguyen KT, Zhao Y, Pathak B, Singh SK (2014) Room temperature chemoselective reduction of nitro groups using non-noble metal nanocatalysts in water. Inorg Chem 53:2904-2909

26. Saravanan P, Jose TA, Thomas PJ, Kulkarni GU (2001) Submicron particles of Co, Ni and Co-Ni alloys. Bull Mater Sci 24(5):515-521

27. Rang HP, Dale MM (1999) Pharmacology, 2nd edn. Churchill Livingstone, Edinburg

28. Begum R, Rehan R, Farooqi ZH, Butt Z, Ashraf S (2016) Physical chemistry of catalytic reduction of nitroarenes using various nanocatalytic systems: past, present and future. J Nanopart Res $18: 231-255$

Publisher's Note Springer Nature remains neutral with regard to jurisdictional claims in published maps and institutional affiliations. 